\title{
Investigação de surto de febre tifóide em Santos, SP, 2004
}

Investigation of an outbreak of typhoid fever in Santos, SP, Brazil 2004

\author{
Divisão de Doenças de Transmissão Hídrica e Alimentar (DDTHA). Centro de Vigilância Epidemiológica \\ "Prof. Alexandre Vranjac". Coordenadoria de Controle de Doenças, Secretaria de Estado da Saúde
}

Em 1/12/2004, foi comunicada, pela Direção Regional de Saúde XIX - Santos, à Divisão de Doenças de Transmissão Hídrica e Alimentar (DDTHA), a ocorrência de dois casos confirmados de febre tifóide (FT), ocorridos no mês de novembro. Entre eles, um óbito em funcionário da Casa de Saúde. Hospital particular e de convênios, no município de Santos, conta com cerca de 100 leitos, unidade de terapia intensiva adulto e neonatal, maternidade e serviços de clínica e cirurgia.

Ações complementares às já iniciadas pelas vigilâncias dos níveis locais - Secretarias Municipais de Saúde de Santos e Guarujá e DIR XIX, foram desencadeadas no mês de dezembro, incluindo-se na investigação a participação conjunta das equipes do CVE (DDTHA, Divisão de Infecção Hospitalar e equipe do Episus-SP); Vigilâncias Epidemiológica e Sanitária dos municípios de Santos e Guarujá e da DIR XIX; Instituto Adolfo Lutz Regional e Central; Divisões de Saneamento e Meio Ambiente e de Alimentos do Centro de Vigilância Sanitária (CVS), bem como da Diretoria Técnica e Comissão de Controle de Infecção Hospitalar (CCIH) da Casa de Saúde de Santos.

Essas ações complementares, visando compreender melhor as possíveis vias de transmissão da doença, foram necessárias pelos seguintes motivos:

1. Trata-se de problema de saúde pública que afeta o nível estadual, uma vez que os casos confirmados e suspeitos de FT notificados pela DIR Santos alteram a tendência epidemiológica, com aumento do número de casos da doença no Estado de São Paulo (Figura 1);

2. A ocorrência de um óbito por FT, que não acontecia desde 1997 (Tabela 1) - o último óbito foi registrado em 1996, no município de Agudos;

3. Atualmente, a FT apresenta baixíssima incidência em São Paulo, com coeficientes em torno de 0,02 por 100 mil habitantes, tornandose uma doença de difícil diagnóstico; assim, o número de casos notificados pode estar apenas representando a ponta do iceberg;

4. São múltiplas suas vias de transmissão - alimentos, água, esgoto, pessoa-a-pessoa, objetos contaminados, fato agravado pela capaci- dade do patógeno em produzir assintomáticos, bem como portadores sãos por longos períodos, ou mesmo permanentes, se não tratados adequadamente. Devido a esta característica, sua investigação é mais complexa e pode exigir, para a identificação de suas causas, além das investigações epidemiológica e sanitária convencionais, estudos e inquéritos epidemiológicos adicionais;

5. Os casos confirmados e suspeitos se concentram em pacientes e funcionários da Casa de Saúde de Santos, residentes em diferentes municípios da DIR.

Dessa forma, nos dias 23/12/2004 e 5/1/2005 as equipes se reuniram nos municípios de Santos e Guarujá para realizar a investigação complementar dos casos, com outras reuniões e ações programadas para o mês de janeiro de 2005 .

\section{Investigação}

A investigação epidemiológica encontra-se em sua primeira etapa, correspondente a estudo descritivo que consta da identificação dos casos, rastreamento de casos suspeitos, portadores e contatos, exames laboratoriais e levantamento dos fatores de risco relacionados ao ambiente hospitalar e à procedência dos casos - bairros e municípios de moradia-,

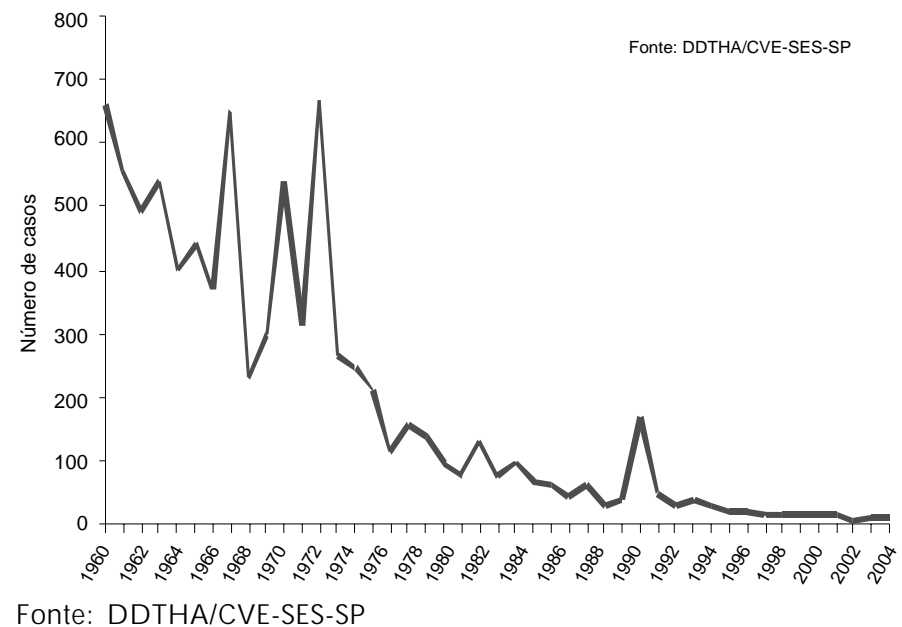

Figura 1 - Febre tifóide. Casos autóctones confirmados. Estado de São Paulo, 1960-2004. 
Tabela 1 - Febre tifóide: óbitos no Estado de São Paulo, 1980-2004.*

\begin{tabular}{lc}
\hline Ano & Ó bitos \\
\hline 1980 & 4 \\
1981 & 2 \\
1982 & 4 \\
1983 & 4 \\
1984 & 1 \\
1985 & 1 \\
1986 & 5 \\
1987 & 4 \\
1988 & 0 \\
1989 & 3 \\
1990 & 4 \\
1991 & 0 \\
1992 & 1 \\
1993 & 0 \\
1994 & 1 \\
1995 & 2 \\
1996 & 1 \\
1997 & 0 \\
1998 & 0 \\
1999 & 0 \\
2000 & 0 \\
2001 & 0 \\
2003 & 0 \\
$2004 *$ & 0 \\
\hline Fonte: SEADE (dados de 1980 até outubro de 2004) \\
*Dados preliminares: 1 óbito por Febre Tifóide detectado \\
através do SVE - DDTHA/CVE-SES-SP \\
\end{tabular}

buscando detectar os elos epidemiológicos entre eles. Inspeções sanitárias têm sido realizadas conjuntamente para avaliação dos fatores de risco para a transmissão da doença, desencadeando-se as ações e medidas de precaução para a interrupção do provável surto.

Para tomar conhecimento das ações já desencadeadas e discutir as hipóteses do provável surto, foram realizadas, inicialmente, reuniões com a presença de todas as instituições anteriormente citadas. Em seguida, foram revisados os prontuários médicos dos dois casos confirmados - funcionários da Casa de Saúde de Santos (internados no hospital do Guarujá e residentes neste município), bem como analisadas as fichas de notificação de pacientes suspeitos e confirmados. Foram, ainda, realizadas visitas domiciliares aos casos confirmados e entrevistas com outros funcionários da Casa de Saúde notificados por suspeita de FT. Também foram verificadas todas as coproculturas e hemoculturas positivas de Salmonella, realizadas pelo laboratório da Casa de Saúde.

Além disso, juntamente com as equipes de vigilância sanitária local e regional, foram realizadas visitas às dependências relacionadas às questões alimentares e de meio ambiente da Casa de Saúde.

Para a vigilância prospectiva de FT entre os funcionários do hospital, a partir de primeiro de janeiro de 2005, definiu-se como caso todo "funcionário da Casa de Saúde de Santos que apresentar febre e quadro abdominal (obstipação, diarréia ou dor abdominal), acompanhados ou não dos seguintes sintomas: náuseas, vômitos, cefaléia, hepatomegalia, esplenomegalia, astenia, mal-estar, roséola tífica (manchas avermelhadas) ou dissociação pulso-temperatura; ou febre de início insidioso e persistente, sem sinais de localização". Para o estudo retrospectivo, com base na revisão de prontuários de pacien- tes e funcionários internados no hospital e verificação dos registros laboratoriais, utilizou-se a mesma definição acima, estendendo-se a análise aos anos de 2003 e 2004.

Cabe ressaltar que essa definição se aplica à notificação em geral de casos suspeitos de FT, sendo a doença de notificação obrigatória e imediata aos órgãos de vigilância epidemiológica local, regional e central.

Os casos confirmados laboratorialmente são aqueles com quadro clínico compatível e isolamento de Salmonella typhi em culturas de sangue, urina ou fezes ou detecção por técnicas de biologia molecular. Os casos confirmados por critério clínico-epidemiológico são aqueles com quadro clínico compatível e epidemiologicamente associado a um caso confirmado por critério laboratorial.

Para o estudo de portadores no hospital, indivíduos que, após enfermidade clínica ou sub-clínica (assintomáticos), continuam eliminando bacilos por vários meses, manipuladores de alimentos ou não, mas vinculados a áreas críticas, com possibilidade de contaminação ambiental ou transmissão pessoa-a-pessoa, serão realizadas coproculturas seqüenciais para a detecção de S. typhi, conforme estabelece o manual de orientações da DDTHA/CVE sobre S. typhi/Febre Tifóide (http:/ /www.cve.saude.sp.gov.br/htm/hidrica/if_510FT.html).

Os exames laboratoriais correspondentes à investigação em curso estão sendo realizados pelo IAL Regional, para isolamento da Salmonella e encaminhados ao IAL Central, para a sorotipagem e outros testes complementares, incluindo-se os de biologia molecular para tentar determinar o perfil genético da bactéria.

\section{Resultados preliminares da investigação}

Até o momento, foram identificados na Casa de Saúde dois casos confirmados laboratorialmente e 10 suspeitos, em 2004 (Tabela 2). Os casos confirmados são residentes do Guarujá e funcionários da Casa de Saúde de Santos, sendo que um deles evoluiu para óbito.

Dos casos suspeitos rastreados na investigação, em sete foi encontrada a Salmonella sp. Entre eles, três funcionários da Casa de Saúde (dois deles S. typhi) e quatro pacientes provenientes da comunidade. Dessa forma, para o ano de 2004, foram obtidos dados de 10 pacientes (dois confirmados e oito suspeitos, sendo seis casos funcionários da Casa de Saúde), apresentados no Tabela 2 e Figura 2. Os três casos restantes, funcionários da Casa de Saúde, notificados em dezembro, encontram-se em investigação laboratorial. Esses casos são procedentes de Santos, São Vicente, Guarujá e Praia Grande.

Para o ano de 2003, já foram identificados mais quatro pacientes com culturas positivas para Salmonella sp., cuja investigação está em andamento.

Todos os casos confirmados ou suspeitos apresentaram febre e náuseas; três desenvolveram hepatoesplenomegalia. 
Tabela 2 - Resumo dos casos suspeitos e confirmados de febre tifóide entre pacientes e funcionários. DIR XIX - Santos, 2004.*

\begin{tabular}{|c|c|c|c|c|c|c|c|c|c|c|}
\hline Iniciais & EJO & ALO & GSB & ESSA & NFGV & EBS & GLB & AASG & ООТВ & $\mathrm{HA}$ \\
\hline Idade & 23 & 32 & 55 & 34 & 16 & 27 & 27 & 28 & 80 & 20 \\
\hline Sexo & $\mathrm{F}$ & $\mathrm{F}$ & $M$ & $\mathrm{~F}$ & $\mathrm{~F}$ & $\mathrm{~F}$ & $\mathrm{~F}$ & $\mathrm{~F}$ & $\mathrm{~F}$ & $M$ \\
\hline $\begin{array}{l}\text { Funcionário/ } \\
\text { paciente }\end{array}$ & $\begin{array}{c}\text { Técnica } \\
\text { enfermagem } \\
\text { maternidade }\end{array}$ & $\begin{array}{l}\text { Camareira } \\
\text { lavanderia }\end{array}$ & $\begin{array}{l}\text { Supervisor } \\
\text { enfermagem }\end{array}$ & $\begin{array}{c}\text { Instrumentadora } \\
\text { CO }\end{array}$ & Paciente & $\begin{array}{c}\text { Auxiliar de } \\
\text { enfermagem } \\
\text { UTI }\end{array}$ & Paciente & $\begin{array}{l}\text { Supervisor } \\
\text { enfermagem }\end{array}$ & Paciente & Paciente \\
\hline Turno/ leito & Noturno & Diurno & Noturno & Diurno & $2^{\circ}(216 / 01)$ & Diurno & $1^{\circ}(114)$ & Diurno & $2^{\circ}(236 / 01)$ & $2^{\circ}(253 / 01)$ \\
\hline DT Sintomas & $27 / 12 / 04$ & $29 / 10 / 04$ & $1 / 12 / 04$ & $17 / 12 / 04$ & $27 / 2 / 04$ & $6 / 4 / 04$ & $20 / 9 / 04$ & $22 / 12 / 04$ & $22 / 12 / 04$ & $25 / 12 / 04$ \\
\hline Diagnóstico & $\begin{array}{c}\text { Abdome } \\
\text { agudo æpse }\end{array}$ & $\begin{array}{l}\text { Dengue } \\
\text { gravidez }\end{array}$ & $\begin{array}{c}\text { GECA } \\
\text { Colecistite } \\
\text { calculosa }\end{array}$ & GECA & Salmonelose & $\begin{array}{l}\text { Salmonelose } \\
\text { anemia }\end{array}$ & $\begin{array}{c}\text { LES } \\
\text { salmonelose }\end{array}$ & GECA & $\begin{array}{c}\text { DM/HAS } \\
\text { GECA }\end{array}$ & GECA \\
\hline Evolução & Óbito & & & & & & & & & \\
\hline Febre & Sim & Sim & Sim & Sim & Sim & Sim & Sim & Sim & Sim & Sim \\
\hline Astenia & Sim & Sim & Sim & Sim & Sim & Sim & Sim & Não & Sim & Sim \\
\hline Náuseas & Sim & Sim & Sim & Sim & Sim & Sim & Sim & Sim & Sim & Sim \\
\hline Cefaléia & Sim & Sim & Sim & Sim & Não & Sim & Sim & Sim & Sim & Sim \\
\hline Vômitos & Sim & Sim & Sim & Sim & Sim & Sim & Sim & Não & Não & Sim \\
\hline Dor abdominal & Sim & Não & Sim & Sim & Sim & Sim & Sim & Sim & Não & Sim \\
\hline Diarréia & Sim & Sim & Sim & Sim & Sim & Sim & Sim & Sim & Sim & Sim \\
\hline Esplenomegalia & Sim & Não & Sim & Não & Não & Sim & Não & Não & Não & Não \\
\hline Obstirpação & Sim & Sim & Não & Não & Não & Não & Não & Sim & Sim & Não \\
\hline Tosse & Não & Não & Não & Não & Não & Sim & Não & Não & Não & Não \\
\hline Perfuração & Sim & Não & Não & Não & Não & Não & Não & Não & Não & Não \\
\hline Outros & $\begin{array}{l}\text { Mialgia, icterícia } \\
\text { choque séptico }\end{array}$ & $\begin{array}{c}\text { Mialgia } \\
\text { anorexia }\end{array}$ & $\begin{array}{c}\text { Mialgia } \\
\text { anorexia }\end{array}$ & $\begin{array}{c}\text { Anorexia } \\
\text { hipotensão } \\
\text { sonolência }\end{array}$ & $\begin{array}{c}\text { Toxemia } \\
\text { hipotensão }\end{array}$ & $\begin{array}{l}\text { Hepatomegalia } \\
\text { hipotensão } \\
\text { icterícia } \\
\text { mialgia anorexia }\end{array}$ & $\begin{array}{l}\text { Toxemia } \\
\text { hipotensão }\end{array}$ & $\begin{array}{l}\text { Mialgia } \\
\text { artralgia }\end{array}$ & $\begin{array}{l}\text { Sonolência } \\
\text { anorexia }\end{array}$ & $\begin{array}{c}\text { Mialgia } \\
\text { hipotensão }\end{array}$ \\
\hline Hemocultura & S. typhi & $\begin{array}{c}\text { Bacilo gram } \\
\text { negativo }\end{array}$ & Negativa & Negativa & $\begin{array}{l}\text { Não } \\
\text { realizada }\end{array}$ & Salmonella sp & $\begin{array}{l}\text { Não } \\
\text { realizada }\end{array}$ & Negativa & $\begin{array}{l}\text { Não } \\
\text { realizada }\end{array}$ & $\begin{array}{l}\text { Não } \\
\text { realizada }\end{array}$ \\
\hline Coprocultura & Não realizada & S. typhi & Negativa & Negativa & Salmonella sp & Salmonella sp & Salmonella sp & Negativa & Salmonella sp & o Salmonella sp \\
\hline $\mathrm{Hb} / \mathrm{Ht}$ & Anemia & Normal & - & - & Normal & Anemia & Normal & - & Normal & Normal \\
\hline Leucócitos & Leucocitose & Leucopenia & - & - & Normal & Leucocitose & Leucocitose & - & Normal & Normal \\
\hline Bast/ segm & Desvio à esq & Desvio à esq & - & - & Desvio à esq & Desvio à esq & Desvio à esq & - & Normal & Desvio à esq \\
\hline Eosinófilos & $0 \%$ & $0 \%$ & - & - & $1 \%$ & $0 \%$ & $0 \%$ & - & $1 \%$ & $1 \%$ \\
\hline Antibiótico & $\begin{array}{l}\text { Amica/Rocefin/ } \\
\text { Cipro/Flagyl }\end{array}$ & $\begin{array}{c}\text { Keflex } \\
\text { Ampicilina }\end{array}$ & Cipro & Cloranfenicol & Ofloxacino & Cipro & Cipro bactrim & Cipro & Cipro & Cipro \\
\hline Duração & 10 dias & 10 dias & 7 dias & 2 dias & $\operatorname{lgn}$ & 10 dias & $\operatorname{lgn}$ & 7 dias & $\lg n$ & $\lg n$ \\
\hline Bairro/ Cidade & $\begin{array}{c}\text { Morrinhos III - } \\
\text { Guarujá }\end{array}$ & $\begin{array}{l}\text { Paecará - } \\
\text { Guarujá }\end{array}$ & $\begin{array}{l}\text { V. Belmiro - } \\
\text { Santos }\end{array}$ & $\begin{array}{c}\text { V. Mirim- } \\
\text { Praia Grande }\end{array}$ & $\begin{array}{l}\text { Boqueirão - } \\
\text { Santos }\end{array}$ & $\begin{array}{c}\text { Jd. Santana - } \\
\text { Guarujá }\end{array}$ & $\begin{array}{l}\text { V. Belmiro - } \\
\text { Santos }\end{array}$ & $\begin{array}{l}\text { V. Valência } \\
\text { São Vicente }\end{array}$ & $\begin{array}{l}\text { Embaré- } \\
\text { Santos }\end{array}$ & $\begin{array}{c}\text { Gonzaga- } \\
\text { Santos }\end{array}$ \\
\hline Refeitório & Sim & Sim & Sim & Sim & Sim & Sim & Sim & Sim & Sim & Sim \\
\hline Cantina & $\lg n$ & Sim & Não & Não & Não & Sim & Não & Não & Não & Não \\
\hline Água de poço & Sim & Sim & Sim & Sim & $\operatorname{lgn}$ & Sim & $\operatorname{lgn}$ & Sim & $\lg n$ & $\lg n$ \\
\hline Ambulantes & $\operatorname{lgn}$ & Não & Não & Não & $\operatorname{lgn}$ & Não & $\operatorname{lgn}$ & Não & $\lg n$ & $\operatorname{lgn}$ \\
\hline Contato & Não & Não & Não & Não & Não & Não & Não & Não & Não & Não \\
\hline
\end{tabular}

Fonte: Div Doenças de Trnsmissão Hídrica e Alimentar/CVE *Dados atualizados até $11 / 1 / 2005$

Quase todos os casos apresentaram cefaléia e astenia (90\%).

A investigação sanitária do ambiente hospitalar encontra-se também em andamento, tendo sido já verificados o sistema de abastecimento e distribuição da água, disposição dos resíduos sólidos, o refeitório, copas e lanchonete, as condições sanitárias de áreas onde trabalham os funcionários que adoeceram, escala de funcionários, contatos entre eles, os protocolos de controle da infecção hospitalar junto à CCIH, dentre outros aspectos, incluindo-se os arredores do hospital, onde os funcionários podem ter feito suas refeições. A vigilância sanitária local tomou várias medidas, além das educativas, interditando um poço artesiano utilizado pelo hospital, por estar em desacordo com as exigências legais.

A investigação das condições sanitárias dos bairros/municípios de procedência dos pacientes (funcionários e não funcionários) também está em andamento, verificando-se, contudo, condições precárias de saneamento básico no bairro de residência de um dos casos confirmados.

A partir desses resultados, algumas hipóteses foram levantadas para explicar os elos epidemiológicos do surto: 1) transmissão por fonte comum, relacionada a alguma depen- 
dência do hospital (refeitório, lanchonete, fontes de água como bebedouro, poço artesiano, torneiras, entre outras); 2) transmissão pessoa-a-pessoa, por meio de doentes ou portadores assintomáticos provenientes da comunidade ou entre funcionários do hospital.

\section{Recomendações}

Com base nos resultados obtidos até o momento, não se pode ainda concluir sobre a via de transmissão, isto é, confirmar ou descartar as hipóteses levantadas, estabelecendo-se ainda várias tarefas conjuntas para a conclusão da investigação, bem como recomendações:

1. Afastamento dos funcionários com suspeita de febre tifóide e de portadores, até o término do tratamento adequado e realização das coproculturas de controle seqüenciais;

2. Reforço às recomendações de boas práticas de higiene no hospital, tais como lavagem de mãos, entre outras, o que é fundamental para bloquear a cadeia de transmissão da doença;

3. Reforço às boas práticas de produção/manipulação de alimentos, tais como práticas de higiene nas cozinhas (refeitório e lanchonete), lavagem das mãos, cuidados com a origem da matéria-prima e de fornecedores (alimentos previamente preparados), desinfecção dos alimentos crus, controle de pontos críticos, refrigeração e armazenamento adequados dos alimentos, paramentos dos funcionários, afastamento de portadores (pesquisa em andamento), dentre outros aspectos;

4. Realização da coleta de exames de coprocultura e hemocultura dos funcionários ou pacientes com quadro clínico compatível com febre tifóide;

5. Análise de biologia molecular (Pulsed Field) dos isolados de $S$. typhi relacionados com o surto pelo IAL Central;

6. Delineamento de um inquérito (estudo descritivo retrospectivo) de pacientes internados na Casa de Saúde nos meses de setembro, outubro e novembro de 2004, para verificar sinais e sintomas compatíveis com febre tifóide nos três meses posteriores à sua alta hospitalar;

7. Levantamento, junto ao Centro de Processamento de Dados do hospital, das variáveis constantes do sistema de informação de internação, para o delineamento posterior do estudo epidemiológico de funcionários e pacientes;

8. Estabelecimento de um fluxo em que todas as cepas de Salmonella isoladas em culturas sejam enviadas ao Instituto Adolfo Lutz para tipagem;

9. Aplicação da ficha elaborada para a vigilância epidemiológica de febre tifóide nos funcionários da Casa de Saúde que preencherem a definição de caso descrita na mesma;

10. Realização de um inquérito retrospectivo entre funcionários dos setores críticos do hospital, bem como dos setores onde ocorreram outros casos suspeitos entre funcionários;

11. Investigação de sintomas gastrointestinais (busca ativa) em pacientes que reinternarem até três meses após a última internação;

12. Revisão dos prontuários dos pacientes com culturas positivas (Salmonella sp.) encontradas nos anos de 2003 e 2004:

13. Avaliação clínica dos 34 funcionários da equipe de enfermagem que cuidaram dos pacientes identificados com culturas positivas para Salmonella sp. em 2004;

14. Levantamento de dados de outras fontes de registro oficiais sobre diarréia e outros quadros gastrointestinais e diagnósticos similares ao quadro de FT (AIH/Datasus, MDDA, VEDTA, etc.);

15. Reforço às investigações das condições sanitárias de ruas e bairros dos municípios onde moram os pacientes confirmados e suspeitos de FT (funcionários e não funcionários), com monitoramento ambiental e outras medidas;

16. Alerta à comunidade médica local e laboratórios para a notificação imediata de casos suspeitos de FT e de Salmonellas detectadas na região da Baixada Santista, com envio dos isolados para o IAL para a tipagem;

17. Alerta à comunidade médica em geral para maior atenção a quadros suspeitos de FT, ficando à cargo da DDTHA/CVE a elaboração de um breve informe técnico para ser divulgado através dos boletins do Conselho Regional de Medicina.

Existem ainda muitas etapas a serem cumpridas nessa investigação, inclusive a possibilidade de se realizar um estudo epidemiológico analítico com os casos (doentes e portadores) detectados a partir do estudo descritivo para a detecção da via de transmissão. O que apresentamos foram os resultados preliminares de um estudo exploratório, para divulgar à comunidade médico-científica o trabalho cooperativo realizado por instituições de diversas instâncias, com o objetivo de estabelecer o controle da cadeia de transmissão da febre tifóide a partir do esclarecimento das causas do surto. 